\title{
Development of a lower extremity wearable exoskeleton with double compact elastic module: preliminary experiments
}

\author{
Yi Long ${ }^{1,2}$, Zhi-jiang Du ${ }^{1}$, Chao-feng Chen ${ }^{1}$, Wei-dong Wang ${ }^{1}$, and Wei Dong ${ }^{1}$ \\ ${ }^{1}$ State Key Laboratory of Robotics and System, Harbin Institute of Technology (HIT), Harbin, China \\ ${ }^{2}$ Zhongshan Torch Group, Co. Ltd, Zhongshan, China \\ Correspondence to: Wei Dong (dongwei@hit.edu.cn)
}

Received: 18 April 2017 - Revised: 28 June 2017 - Accepted: 12 July 2017 - Published: 14 August 2017

\begin{abstract}
In this paper, a double compact elastic module is designed and implemented in the lower extremity exoskeleton. The double compact elastic module is composed of two parts, i.e., physical human robot interaction (pHRI) measurement and the elastic actuation system (EAS), which are called proximal elastic module (PEM) and distal elastic module (DEM) respectively. The PEM is used as the pHRI information collection device while the DEM is used as the compliance device. A novel compact parallelogram-like structure based torsional spring is designed and developed. An iterative finite element analysis (FEA) based optimization process was conducted to find the optimal parameters in the search space. In the PEM, the designed torsional spring has an outer circle with a diameter of $60 \mathrm{~mm}$ and an inner hole with a diameter of $12 \mathrm{~mm}$, while in the DEM, the torsional spring has the outer circle with a diameter of $80 \mathrm{~mm}$ and the inner circle with a diameter of $16 \mathrm{~mm}$. The torsional spring in the PEM has a thickness of $5 \mathrm{~mm}$ and a weight of $60 \mathrm{~g}$, while that in the DEM has a thickness of $10 \mathrm{~mm}$ and a weight of $80 \mathrm{~g}$. The double compact elastic module prototype is embedded in the mechanical joint directly. Calibration experiments were conducted on those two elastic modules to obtain the linear torque versus angle characteristic. The calibration experimental results show that this torsional spring in the PEM has a stiffness of $60.2 \mathrm{Nm} \mathrm{rad}^{-1}$, which is capable of withstanding a maximum torque of $4 \mathrm{Nm}$, while that in the DEM has a stiffness of $80.2 \mathrm{Nm} \mathrm{rad}^{-1}$, which is capable of withstanding a maximum torque of $30 \mathrm{Nm}$. The experimental results and the simulation data show that the maximum resultant errors are $6 \%$ for the PEM and $4 \%$ for the DEM respectively. In this paper, an assumed regression algorithm is used to learn the human motion intent (HMI) based on the pHRI collection. The HMI is defined as the angular position of the human limb joint. A closed-loop position control strategy is utilized to drive the robotic exoskeleton system to follow the human limb's movement. To verify the developed system, experiments are performed on healthy human subjects and experimental results show that this novel robotic exoskeleton can help human users walk, which can be extended and applied in the assistive wearable exoskeletons.
\end{abstract}

1

\section{Introduction}

Wearable exoskeletons can enhance the power of operators to assist walking or carrying heavy loads. The robotic exoskeleton has the similar mechanical structure with the human limb. In recent years, many advances and progress have been made in the development of wearable exoskeletons. In 2000, the DARPA sponsored the program, i.e., Exoskeletons for $\mathrm{Hu}-$ man Performance Augmentation (EHPA), which aimed to developing several kinds of wearable exoskeletons to increase and improve the soldiers' capabilities, e.g., BLEEX, ExoClimber, ExoHiker and HULC (Kazerooni and Steger, 2006). In the field of wearable robots for assistance and rehabilitation, Hybrid Assistive Limb (HAL), developed by the University of Tsukuba in Japan, is utilized to help the operator walk and carry heavy loads (Sankai, 2010; Kawamoto et al., 2003). The wearable exoskeleton robot is a typical human-robot cooperation system, where the human operator 
is located in the control loop. Therefore, in order to achieve human exoskeleton cooperation, the most important step is to acquire and estimate the HMI via measuring the HRI. According to the measurement methodologies, the HRI signals can be divided into three kinds of classification, i.e., biomedical signals, e.g., Electromyographic (EMG), physical HRI signals, e.g., force or torque signals, and mechanical signals only from exoskeletons themselves (Huo et al., 2016). The biomedical signal, e.g., sEMG, is used widely in exoskeletons for power assistance or rehabilitation (Lee and Sankai, 2002). However, the function of the sEMG will be affected by the individual difference and the skin surface conditions. The HRI measured by mechanical sensors on the exoskeleton uses states and feedback signals of the system. However, this kind of signal needs an accurate mathematical model and is sensitive to external disturbances (Kazerooni et al., 2005). The pHRI collection is adopted by some exoskeletons, e.g., WPS, developed by Kanagawa Institute of Technology (Dollar and Herr, 2008; Yoshimitsu and Yamamoto, 2004). In this way, sensors are generally placed on the interface between the exoskeleton and the operator.

The pHRI information can be obtained by force or torque sensors. A flexible sensor technology is developed to measure the pHRI pressure, where the sensory system is composed of several optical-electronic sensors (Donati et al., 2013). The system has at least six sensors at the interaction cuff. The pHRI force can be measured by a strain gauge, where a circle sensor is utilized (Del-Ama et al., 2012). The wearing comfort can be ensured by the human robot interfaces to achieve the physical and cognitive cooperation. The connection cuff is a widely-used way to connect the wearable robot and the human user. It is critical to get an accurate measurement of the pHRI information for the robotic exoskeleton control and the assistance grade evaluation (Santis et al., 2008). The pHRI signal can be measured by estimating the joint torque of exoskeleton, e.g., LOPES (Veneman et al., 2007) and directly measured by using force sensors or torque sensors (Lee et al., 2008). The first approach needs an accurate HRI dynamics model and the second one leads to a complex structure with many sensors (Lenzi et al., 2011). The pHRI information measured by sensors placed on the connection cuff is capable of representing the interaction directly. This type of measurement utilizes one sensor or several sensors. The usage of one single sensor can make the structure compact but may hide necessary information, while the usage of several sensors can measure the HRI more completely but results in a complicated structure. The multi-sensor device, called pressure sensor pads, is applied for robotic rehabilitation (De Rossi et al., 2010a, b). Those sensors are placed on the connection cuff, where the tightness has an important effect on the accurate measurement, besides, the cuff with embedded sensors may cause the operator's wearing discomfort. Similarities of those reported methods are presented as the follows, i.e., (1) sensors are placed on the interaction cuff, (2) multi-sensor system will complicate the structure of the interface.

Different from that presented previously, this paper proposes a compact measurement structure placed on the robot joint directly instead of in the connection cuff. The measurement structure is dependent on an elastic module, which is composed of tensional springs and magnetic encoders. The relative movement will cause the elastic module's deformation and the magnetic encoder will output the measurement signal. This kind of pHRI measurement only needs an encoder and simplifies the structure of the connection cuff. Considering the human limb's softness, the sensor for pHRI measurement should has high resolution without causing the wearing discomfort. In this paper, a rotary torsion spring is developed and connected with a position sensor, which is tolerant of shocking to ensure the wearing comfort.

In reality, this kind of elastic module is inspired by the serial elastic actuator (SEA) in the wearable exoskeleton. In the SEA, the elastic element is installed between the output of the motor and the carrying load. The SEA is adopted in the robotic exoskeleton due to its several benefits (Arumugom et al., 2009). Compared with an ordinary stiff actuator, the SEA has more advantages in following features, i.e., lower output impedance and back-drivability, shock tolerance, force transmission fidelity, storing and releasing energy, and passive impedance at high frequencies. Accoto et al. presented a kind of SEA for a lower limb robotic orthosis, where a custom-made torsion spring with a stiffness of $272.25 \mathrm{Nm} \mathrm{rad}^{-1}$ is directly connected to the load and the delivered torque can be obtained by measuring the deflection of the spring through absolute encoders (Accoto et al., 2013). Several kinds of elastic springs in SEA were concluded and presented that linear springs and torsional springs were arranged in the transmission train actuators, where the discrete design details are described (Carpino et al., 2012). Considering the specialty of robot joint structure, the spring structure is usually rotary. A compact series elastic actuator is designed to satisfy those requirements, e.g., back drivability and low output impedance, and the actuation performance is verified by experiments (Kong et al., 2012). Hao et al. (2017) made much progress in the field of elastic actuators for the flexible mechanisms and have given the detailed analysis for the elastic actuators' compliance (Hao et al., 2017; Hao, 2017a, b). However, those torsional springs are only used in SEA. In the assistive exoskeletons, acquiring the HMI is a crucial issue. In this paper, the double elastic module is designed and developed for the lower extremity exoskeleton, where the PEM with the lower stiffness is used for the measurement of the pHRI and the DEM is utilized as the torque sensor for the elastic actuator. Those two elastic modules are used respectively to detect the human movement and enable the mechanical device to move smoothly.

This paper presents the exoskeleton leg with double elastic module, where the PEM is used to measure pHRI information and the DEM is utilized for the elastic actuation system. 
Based on the discussion above, we highlight the following features,

1. A novel double elastic module is designed and implemented for the lower extremity exoskeleton to help the human user walk. The proposed double elastic module is composed of the PEM and the DEM.

2. The developed double elastic module is placed on the mechanical joint. It has two main functions, i.e., measuring the pHRI information and acting as the torque sensor. Compared with the traditional pHRI measurement where the utilized sensors are placed at the interaction cuff, the measurement device is capable of freeing the human leg and increasing the wearing comfort.

3. Compared with the regular SEA, the proposed mechanism has the similar functions and furthermore can detect the human movement intention directly.

\section{FEA-based design of torsional spring}

\subsection{Design process of torsional spring}

Since the torsional springs have the similar structure in PEM and DEM, the PEM's spring is introduced as example to present the design process and performance analysis in this paper. In robotic exoskeleton, the pHRI information is generated when the human user wants to move. Some mechanical sensors are designed and developed to measure the pHRI. Since the high accuracy and stability of pHRI collection has an important influence on the estimation of the human motion intent, the elastomer is the key element in the design of sensors. The mechanical design and performance analysis are presented in this section. The elastic module is designed to collect the pHRI, which focuses on the achievement of two objectives, i.e., (1) the capability of measuring the HRI with high resolution, (2) the minimization of weight and dimensions. The maximum torque of human exoskeleton interaction is defined as $4 \mathrm{Nm}$. The resolution of the sensor is defined as $0.1 \mathrm{Nm}$. A more compliant component allows a smaller quantization and improves the signal estimation's accuracy. In order to guarantee an accurate torque estimation and consequent performance, a high linearity of the torque versus the angle is desirable. For this same reason, residual deflection (at zero delivered torque) and hysteresis should be avoided (Kong et al., 2012).

To detect the HMI easily and effectively, the elastic module should have high sensitivity and prevent disturbances. Based on those elastic modules in the SEA applied in robotic exoskeleton, a combination of elastic modules structure is constructed. Since the elastic module is arranged on the robot joint, the structure is composed of the outer ring and inner ring, which are connected by the torsional spring to keep the compliance. Its DOF is limited in the proximal plane, which means the structure can rotate clockwise or counterclockwise
Table 1. Design requirements for the elastic module.

\begin{tabular}{ll}
\hline Parameters & Values \\
\hline Stiffness & $65.2 \mathrm{Nm} \mathrm{rad}^{-1}$ \\
Diameter of outer circle & $60 \mathrm{~mm}$ \\
Diameter of inner circle & $8 \mathrm{~mm}$ \\
Maximum torsion torque & $4 \mathrm{Nm}$ \\
Thickness & $5 \mathrm{~mm}$ \\
Maximum deflection & $0.087 \mathrm{rad}$ \\
Minimum deflection & $0.002 \mathrm{rad}$ \\
\hline
\end{tabular}

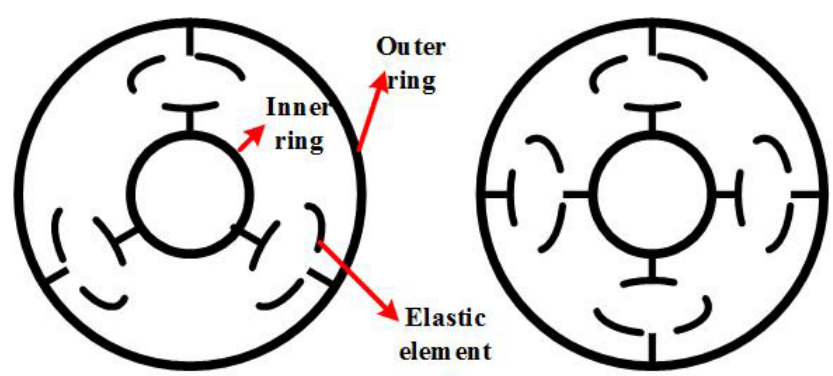

(a)

(b)

Figure 1. Two kind of topologies of elastic modules.

along with the mechanical joint. The specific parameters of the elastic module can be shown in Table 1. The torque applied on the elastic module will be transferred between the outer ring and the inner ring.

The process of the elastic module is presented as follows: (1) a set of topologies determination, (2) mechanical design by using SOLIDWORKS (2012, Dassault Systemes S.A, USA), (3) importing the CAD file into ANSYS (ANSYS 2014, USA), (4) design requirements based simulation. In the iterative FEM of ANSYS, static performance is analyzed to test the stiffness and the linearity. Through the optimization and the selection, the optimal structure of elastic module can be obtained. In this section, two kinds of the elastic element's topologies with symmetric structure are taken into account, which are shown in Fig. 1. Those symmetrical structures have two features, i.e., less optimized parameters and low processing costs. Based on the results presented in Kong et al. (2012), the topology of A is superior to that of B.

The symmetrical structure does not take consideration of the architecture of spiral or double helix, which have backlash such that stiffness and linearity of the elastomer cannot be guaranteed. In Fig. 1, the outer circle is fixed and the inner circle can rotate around the outer circle under the applied torque. A definite topology can be determined by the number and the arrangement of the elastic elements. The definite topology's shape can be defined by a parameter space, e.g., length, width, thickness, and radius. The variable parameters are defined for this topology's specific architecture. The number of variable parameters is $n$ and the range of $i$ th pa- 


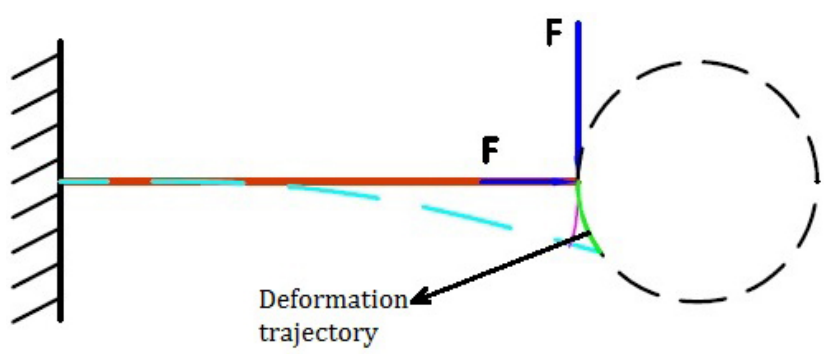

Figure 2. The deformation structure of the beams driven by a hybrid combination of force.

rameter is $\left(x_{i, \min }, x_{i, \max }\right)$, based on which, a $n$-dimensional search space can be determined.

\subsection{Design and simulation of the elastic module}

Based on the topology architecture, the inner circle will rotate around the outer circle under the actuating torque. Generally, the elastic module is actuated by a rotating torque, which can be equivalent to cantilever model that subjects to a force at the free end. The beam will be deformed under the applied force and has deformation in definite direction, as shown in Fig. 2. The deformation of the elastic module can be regarded as a cantilever beam. The tip of the elastic element, fixed with the outer ring, is equivalent to the cantilever's fixed end, while the other tip, fixedly connected with the inner circle, is equivalent to the cantilever's free end. The torque applied to the inner circle is equivalent to the force on the cantilever's free end. In this kind of elastic module, the deformation is caused by the bending and the tensile. The tensile deformation is more obvious. The deformation of tensile can be obtained as the following

$\Delta l=F / E A$

where $\Delta l$ is the deformation along the axial direction, $E$ is Young's modulus, and $A$ is the cross section area. Since $E$ is large, the deformation $\Delta l$ is too small to be measured. Based on the design requirements of dimension and stiffness, the elastic element's deformation cannot be obtained via the tensile deformation along the axial direction while can be achieved by the deformation along the radial direction.

\subsection{Design result of torsional spring}

As discussed previously, the elastic element' deformation can be obtained along the radial direction through a parallelogram-like structure. The radial deformation of this type of structure will be transferred as the bending deformation. Flexible elements are shaped as the arched lamellae placed between the inner circle and the outer circle. $D_{1}$ and $D_{2}$ present the diameter of the outer and inner rings, $D_{3}$ and $D_{4}$ are the distance of the near side and the distal end from the center, $W_{1}$ and $W_{2}$ define the width of the lamellae and

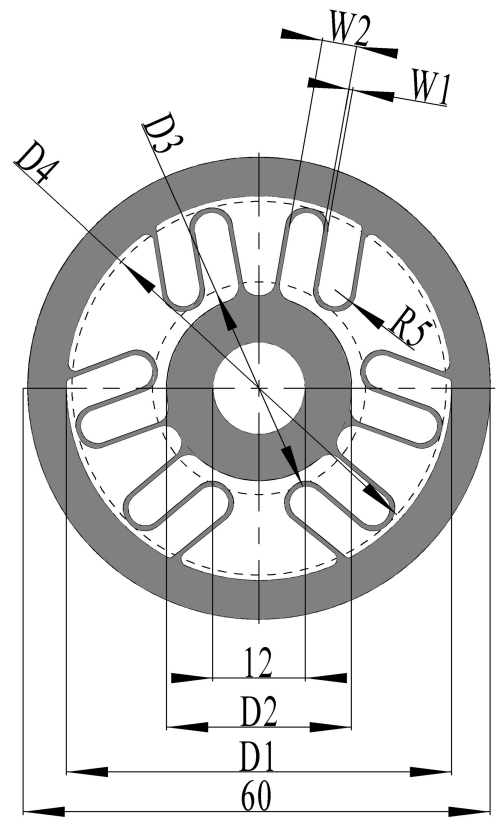

Figure 3. The designed parallelogram-like structure based elastic element.

Table 2. Search space for the optimization of the proposed parallelogram-like topology structure.

\begin{tabular}{lrrr}
\hline Parameter & Minimum & Maximum & Increment \\
\hline$D_{1}$ & 45 & 50 & 1 \\
$D_{2}$ & 24 & 27 & 1 \\
$D_{3}$ & 47 & 48.5 & 0.5 \\
$D_{4}$ & 27 & 29 & 0.5 \\
$W_{1}$ & 0.2 & 1 & 0.1 \\
$W_{2}$ & 3 & 5 & 0.5 \\
$R_{5}$ & 1.5 & 2.5 & 0.05 \\
\hline
\end{tabular}

Notation: the unit of all variables is $\mathrm{mm}$.

the distance between edges of the lamellas and $R_{5}$ is the radius of the lamellae. The thickness of the spokes set, the inner circle's radius and the deformation spokes' distance among three elastic units are set as the same. In order to ensure that the elastic element deforms in the predefined range and those three units do not interfere with each other, the parameters of the elastic element should be located in the specific range, as depicted in Table 2. Table 2 gives detailed illustrations of the design parameters, based on which, the search space can be obtained with the lower and the upper bound as well as the increment of each parameter. The diameters of the inner hole and the outer ring are defined as the constant parameters, i.e., 8 and $60 \mathrm{~mm}$ respectively.

Based on the parallelogram-like structure and the search space defined in Table 2, the optimized structure can be obtained. This type of configuration, as shown in Fig. 3, includes three replications of two couples of parallelogram- 
(a)

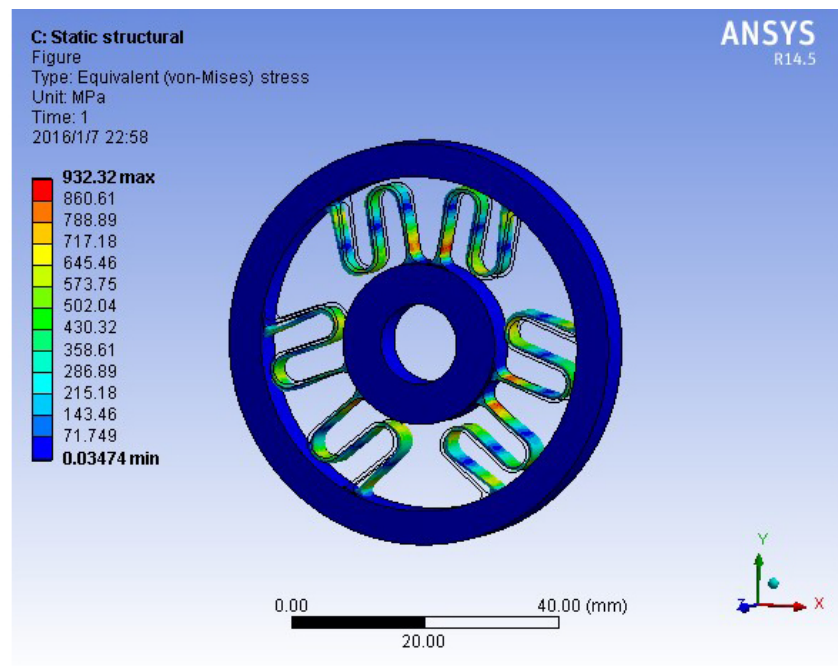

(b)

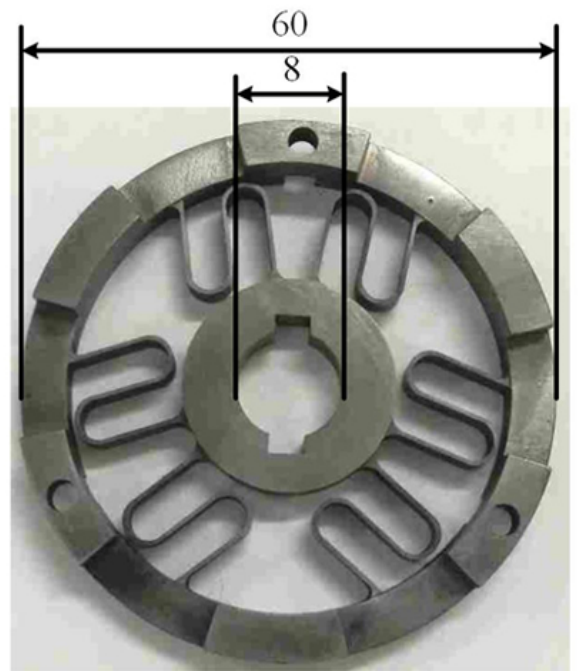

Figure 4. (a) FEM in ANSYS. The deflection with different level applied torque is depicted. (b) The frontal view of the optimized torsional elastic module. The outer diameter: $60 \mathrm{~mm}$, the inner hole diameter: $8 \mathrm{~mm}$, Weight: $60 \mathrm{~g}$.

Table 3. Specific parameters of the optimized elastic element.

\begin{tabular}{lrrrrrrr}
\hline Parameter & $D_{1}$ & $D_{2}$ & $D_{3}$ & $D_{4}$ & $W_{1}$ & $W_{2}$ & $R_{5}$ \\
\hline Value $(\mathrm{mm})$ & 50 & 24 & 48.5 & 27.5 & 0.5 & 45 & 2.25 \\
\hline
\end{tabular}

like lamellae, arranged at $2 / 3 \pi$. There are six elastics parallelogram-like lamellae utilized between the outer circle and the inner circle. In the search space, the final architecture with optimized parameters is obtained after 18 iterations. The diameter of the outer circle is $60 \mathrm{~mm}$. Specific parameters of the designed elastic module are depicted in Table 3. The elastic module can bear the torsion torque up to $4 \mathrm{Nm}$ and the torsion angle can be up to $5^{\circ}$.

Based on FEM, parametric design in the search space for the determined topology structure is conducted. In the search space, the optimized structure is obtained. With the optimized parameters in Table 3, this kind of topology module can be driven by the maximum torque of $4 \mathrm{Nm}$. The $1: 1$ elastic module deformation and the Von Mises Stress plot is shown in Fig. 4a. Based on the FEM in ANSYS, the real sample piece is depicted in Fig. 4b. The optimized module is manufactured by $60 \mathrm{Si} 2 \mathrm{Mn}$ (quenching at $870^{\circ} \mathrm{C}$, oil cooling at $400^{\circ} \mathrm{C}$, Young modulus of $206 \mathrm{GPa}$, Poisson's ratio of 0.59 , yield stress of $1176 \mathrm{Mpa}$, and stretching limit of $1274 \mathrm{Mpa})$. The inner hole and the outer ring of the optimized module are connected with the encoder and the interface with human respectively. After the design optimization, the final torsional spring in the PEM can be obtained, whose properties are presented in Table 4 .

\section{Application in the wearable exoskeleton}

\subsection{Characteristic of the elastic module}

The designed double elastic module is used for the pHRI measurement and also utilized as the elastic actuator. The compliant component of the pHRI measurement has low stiffness to ensure that the pHRI can be collected easily when the operator wears the robotic exoskeleton, while that used for actuation has high stiffness to make sure that the movement is flexible meanwhile bears heavy loads. The robotic exoskeleton is illustrated in Fig. 5 (Long et al., 2017a). In Fig. 5, the left elastic module is used for the pHRI measurement and the right one is utilized for the elastic actuation. The elastic module will be connected with the magnetic encoder. The compact encoder can be implanted into this type of architecture and is fixed to the inner ring. If a torque is applied on the elastic module, the encoder will output corresponding values to reflect the torque. The explosive view of the double elastic module is depicted in Fig. 6. The outer ring is connected with the interaction cuff while the inner ring is fixed on the joint. The deformation of the elastic module can be denoted by the output of the magnetic encoders.

The elastic module's static characterization can be obtained by calibration experiments, where the PEM is conducted as a typical example. The calibration platform can be illustrated in Fig. 7. The inner ring is fixed on a stable table and the encoder is mounted on the outer ring. The external force will produce torque on the torsional spring through the force-bearing bar. The hardware prototype can be shown in Fig. 8, in which the experiment is processed on a stable test bed with bolt connection. The magnetic encoder is connected with the elastic element. The signal of the encoder is collected by a Programmable Multi Axis Controller (PMAC, 


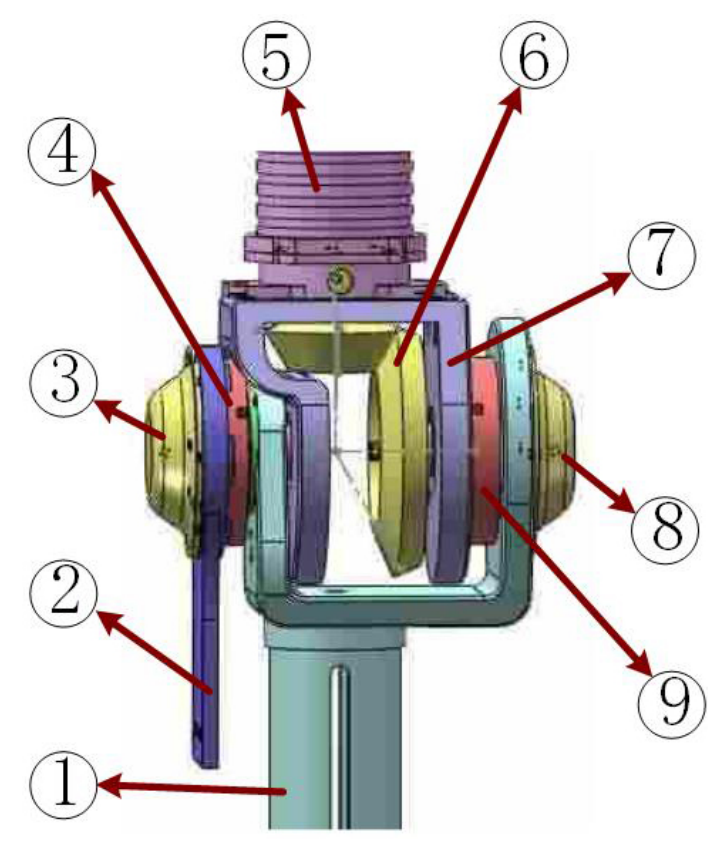

Figure 5. Configuration of the double compact elastic module. 1: the shank segment, 2: the interaction cuff, 3: encoder measuring the position of elastic element, 4: the elastic element of PEM, 5: the thigh, 6: bevel gear pair, 7: the connection part, 8: the encoder, 9: the elastic element of DEM.

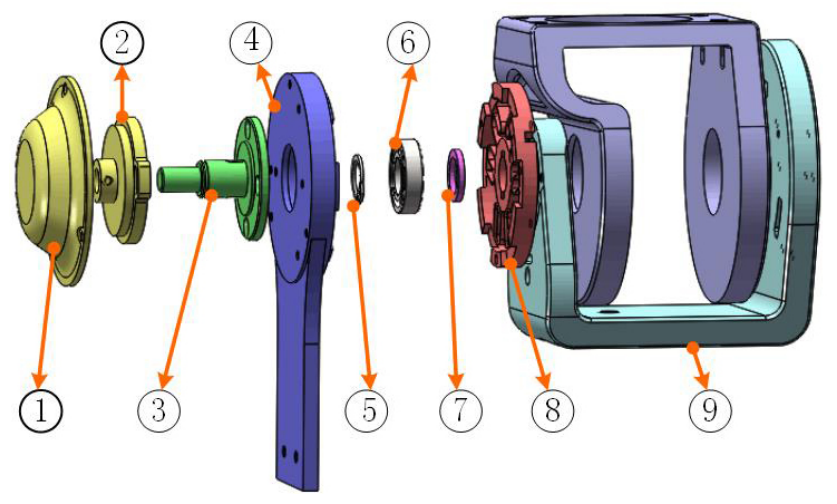

Figure 6. Explosive view of the elastic module. 1: the enclosure, 2: the magnetic encoder, 3 : the encoder support, 4 : the connection cuff, 5:the snap spring, 6: the bearing, 7: the shaft sleeve, 8: the torsional spring, 9: the limb joint.

Delta, USA). The PMAC transfers the position data into the embedded PC through Ethernet. The standard test weights are used to apply force to the torsional elastic module. In Fig. 8, the force limb $D$ is $10 \mathrm{~mm}$, thus the maximum load weight is $4 \mathrm{~kg}$. The minimum weight unit is $50 \mathrm{~g}$ and the maximum weight unit is $2 \mathrm{~kg}$. The load cell by the standard weight changes from $100 \mathrm{~g}$ to $2.5 \mathrm{~kg}$. The increment of load is $50 \mathrm{~g}$. Five tests have been performed for each value of the load weight. The load weight can be removed for each test to allow the element to recover the unperturbed posi-

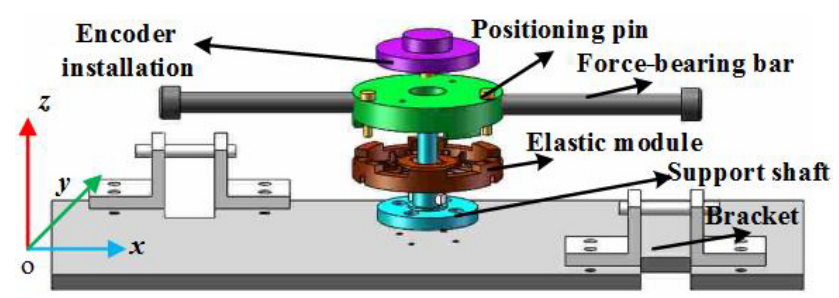

Figure 7. The calibration platform.

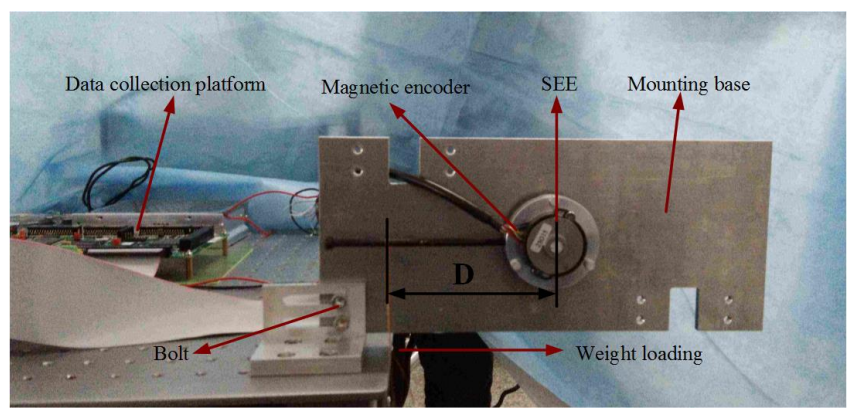

Figure 8. Experiment protocol of the performance verification.

tion. The resulting values are chosen by $95 \%$ confidence. The simulation and experiment data are plotted to illustrate the relationship of torque verse angle as shown in Fig. 9. The FEM simulations data give a prediction of stiffness of $K_{\mathrm{S}}=57.6 \mathrm{Nm} \mathrm{rad}^{-1}$ while the measurements indicate a stiffness of $K_{\mathrm{e}}=60.2 \mathrm{Nm} \mathrm{rad}^{-1}$. Hence, the error between simulation data and experiment data can be obtained according to the following equation

$\operatorname{Err}=\left(K_{\mathrm{e}}-K_{\mathrm{s}}\right) / K_{\mathrm{s}} \times 100 \%$

Therefore, the calculation error is $4.5 \%$.

\section{2 pHRI measuring and processing}

The developed PEM is used to measure the pHRI for wearable exoskeletons. To verify the proposed approach and the developed architecture, the pHRI measurement experiment in the laboratory is conducted on the human upper limb of a healthy subject and ethical approval was granted by the Laboratory Management Council. The elastic module is placed on the joint of human upper limb through connection cuff, as shown in Fig. 10. The elastic module is fixed on a stable platform and is parallel with the elbow joint of the human subject. The upper arm keeps stable as far as possible and the forearm moves to apply torques on the elastic module. The magnetic encoder will register the deformation of the elastic module. The obtained data is angular position (rad), which will be transferred as the torque data $(\mathrm{Nm})$ via stiffness. Five tests are performed and the average of data can be obtained with $95 \%$ confidence. The final experiment result is depicted in Fig. 11. The pHRI curve is split into two parts, i.e., positive and negative direction. The maximum pHRI is smaller 


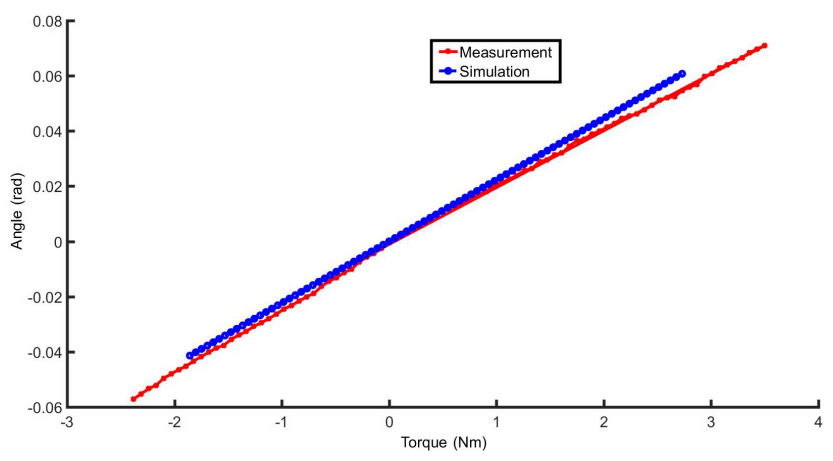

Figure 9. Calibration experiment result. Simulation data are represented with circle dots and experimental data are represented with star dots.

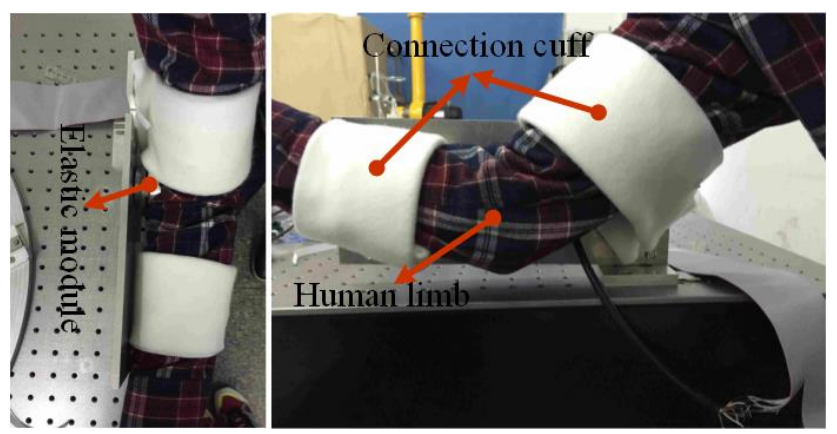

Figure 10. HRI measurement experiment on human upper limb. The connection is used to fasten the elastic module on the human limb. The signals of the magnetic encoder are transferred back to the PC through the PMAC.

than the maximum torque that the elastic module can bear. The pHRI becomes larger when the human user limb feels quite uncomfortable. The pHRI data measured by the elastic module can be utilized in the HMI estimation. The elastic module is capable of measuring the pHRI between the wearable robot and the human operator.

\section{Motion intent-driven control strategy design}

The pHRI can be collected by the PEM in real time. The HMI in this work is defined as the angular position of the human limb joint. The HMI can be reflected and derived by the pHRI. However, the relationship between the pHRI and the HMI cannot be modeled by mathematical approaches easily and directly.

Remark 1: The robotic exoskeleton is a typical highly human-machine coupled system. When the magnitude of the pHRI increases, the corresponding human movement should be quicker. The collected pHRI can reflex the HMI directly. The HMI can be assumed as the function of the pHRI. The magnetic encoder of the PEM can measure the deformation of the torsional spring. Based on the assumption discussed

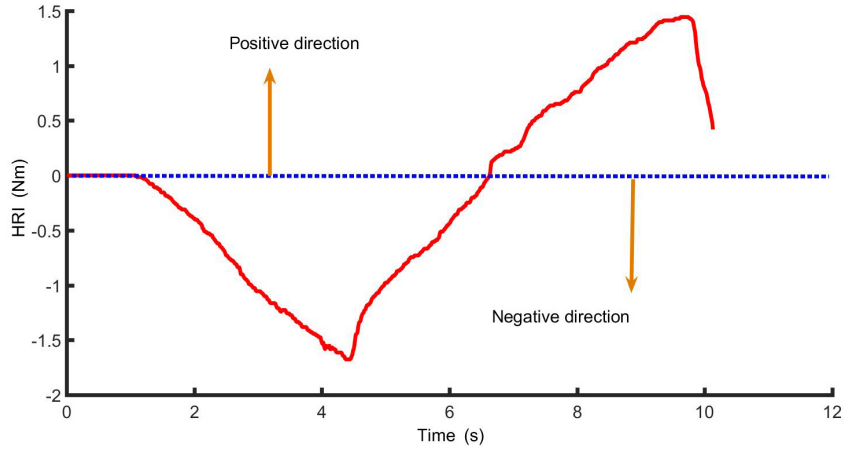

Figure 11. HRI measurement with respect to time. If the torque is zero, the output HRI with the elastic module is close to zero. When the torque drives the elastic module, the HRI will change between the positive interval and the negative interval.

Table 4. Properties of the PEM.

\begin{tabular}{ll}
\hline parameters & values \\
\hline stiffness & $60.2 \mathrm{Nm} \mathrm{rad}^{-1}$ \\
diameter of outer circle & $60 \mathrm{~mm}$ \\
diameter of inner circle & $12 \mathrm{~mm}$ \\
maximum torsion torque & $4 \mathrm{Nm}$ \\
thickness & $5 \mathrm{~mm}$ \\
\hline
\end{tabular}

above, the desired angular position of the mechanical joint can be expressed as follows,

$\theta_{\mathrm{d}}(k)=K \times \theta_{\mathrm{s}}(k)+\theta_{\mathrm{d}}(k-1)$

where, $K$ is a positive constant, $\theta_{\mathrm{d}}(k)$ and $\theta_{\mathrm{d}}(k-1)$ is the $k$ th and $(k-1)$ th desired angular position for the mechanical joint respectively, $\theta_{\mathrm{s}}(k)$ is the deformation of the torsional spring of the PEM. $\theta_{\mathrm{d}}(k)$ will be regarded as the mechanical leg's tracking target.

Remark 2: The desired angular joint of the mechanical leg can be obtained by Eq. (3). The collected $\theta_{\mathrm{s}}(k)$ lags behind the real human motion intent. The time series of $\theta_{\mathrm{s}}(k)$ should be predicted forward to eliminate the delay to improve the wearing comfort.

The HMI can be obtained by the collected pHRI, expressed in Eq. (3). Generally, the measured pHRI include noises at higher frequency. The pHRI should be processed by low-pass filters to eliminate its noises before it is used in Eq. (3). Compared with the biomedical signal, the measured pHRI lags behind the real human motion intention. The delay of pHRI will cause the exoskeleton system not to react to the human limb gait immediately. Overall, the processing steps should include two steps, i.e., (1) eliminating noises and (2) predicting forward. In this paper, Kalman smoother is used to deal with the delay. The general state-space representation of a linear time-variant system is written in the following form 
(Long et al., 2016a; Chen, 2012)

$$
\left\{\begin{array}{l}
\boldsymbol{x}_{k}=\mathbf{A} \boldsymbol{x}_{k-1}+\mathbf{B} \boldsymbol{u}_{k-1}+\boldsymbol{Q}_{k-1} \\
\boldsymbol{y}_{k}=\mathbf{H} x_{k}+R_{k}
\end{array}\right.
$$

where $\boldsymbol{x}_{k}, \boldsymbol{y}_{k}$ and $R_{k}$ are the $k$ th system state, measured output value and the measurement noise vector respectively, $u_{k-1}$ and $\boldsymbol{Q}_{k-1}$ represent the $(k-1)$ th input to the system and system state noise vector, $\mathbf{A}, \mathbf{B}$ and $\mathbf{H}$ describe the state matrix, the input matrix and the output matrix. The primary goal of Kalman filter is to minimize the gap between predicted value and actual value of the target by the least squares method to achieve the optimal results. The updated equation for the system and the measurement equation are shown as follows,

$$
\left\{\begin{array}{l}
\hat{\boldsymbol{x}}_{k}^{-}=\mathbf{A} \hat{\boldsymbol{x}}_{k-1}+\mathbf{B} \boldsymbol{u}_{k-1} \\
\mathbf{P}_{k}^{-}=\mathbf{A P}_{k-1} \mathbf{A}^{\mathrm{T}}+\boldsymbol{Q}_{k-1} \\
\mathbf{K}_{k}=\mathbf{P}_{k}^{-} \mathbf{H}^{\mathrm{T}}\left(\mathbf{H} \mathbf{P}_{k}^{-} \mathbf{H}^{\mathrm{T}}+R_{k}\right) \\
\hat{\boldsymbol{x}}_{k}=\hat{\boldsymbol{x}}_{k}^{-}+\mathbf{K}_{k}\left(\boldsymbol{y}_{k}-\mathbf{H} \hat{x}_{k-1}\right) \\
\mathbf{P}_{k}=\left(\mathbf{I}-\mathbf{K}_{k} \mathbf{H}\right) \mathbf{P}_{k}^{-}
\end{array}\right.
$$

where $\mathbf{K}_{k}$ is the Kalman gain matrix, $\mathbf{I}$ is the unit matrix, $\mathbf{P}_{k}$ is the variance matrix. The Kalman filter only focuses on the measurement data before and at the time step $k$. In this work, Eq. (5) is the system equation of the PEM. Those parameters can be defined as

$$
\begin{gathered}
\mathbf{I}=\left[\begin{array}{ll}
1 & 0 \\
0 & 0
\end{array}\right], \mathbf{A}=\left[\begin{array}{ll}
0 & 1 \\
1 & 0
\end{array}\right], \mathbf{H}=\left[\begin{array}{ll}
1 & 0
\end{array}\right], \\
R=3, \mathbf{Q}=\left[\begin{array}{ll}
0.1 & 0 \\
0 & 0.1
\end{array}\right]
\end{gathered}
$$

The Kalman filter can predict $\theta_{\mathrm{S}}(k)$ to avoid the delay of the time series.

Remark 3: In the preliminary stage, a close loop position strategy is designed to drive the exoskeleton system to follow the human limbs. Once the PEM detect the movement of the torsional spring, the controller will react quickly. The control diagram is depicted in Fig. 12. In the preliminary experiment, a close loop control strategy, called proportion-integrationdifferentiation (PID) control, is designed to drive the robotic exoskeleton to follow the estimated human motion intent.

\section{Experiments for the wearable exoskeleton}

\subsection{Experiment setup}

The control enclosure has the necessary auxiliary facilities, i.e., embedded PC, PMAC, actuators (Copley) and power module. The power supply produces 5,12 and $24 \mathrm{~V}$ voltage. PMAC is used to collect the actual angular position of the joints and execute motion commands from the embedded PC. The magnetic encoders of the double elastic module transferred the elastic torsional springs' deformation to

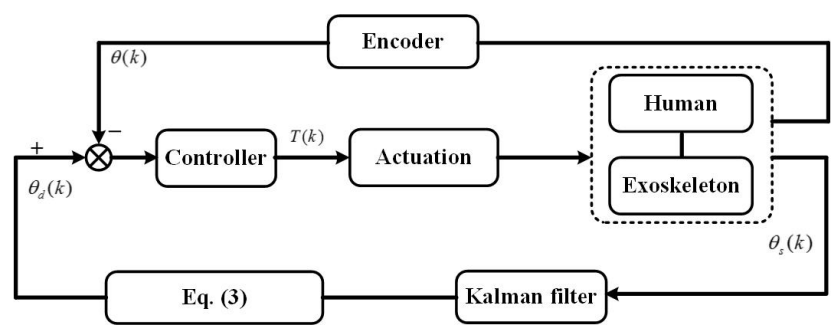

Figure 12. The control diagram for the lower extremity exoskeleton.

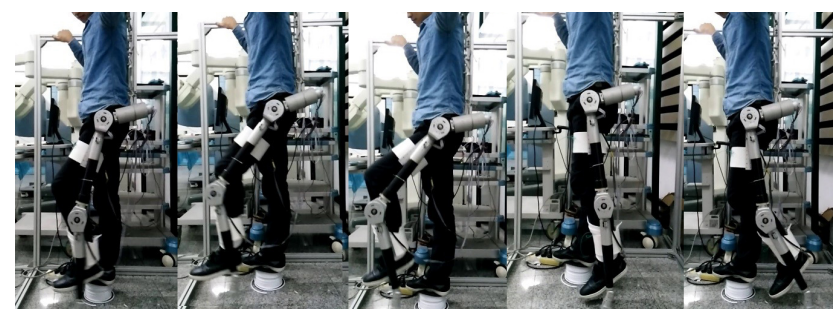

Figure 13. Experiments wearing the lower extremity for the swing leg.

the PC. Since the robotic exoskeleton only has a leg right now, the experiment is conducted for the swing phase. The mechanical leg is driven to follow the human user's swing leg. Three subjects have an average height of $1.75 \pm 0.064 \mathrm{~m}$, an average weight of $60.5 \pm 7.2 \mathrm{~kg}$ and an average age of $26 \pm 1.5$ years old. Safety precautions are taken consideration from the hardware and the software (Long et al., 2016b, 2017b). In the mechanical leg, mechanical limits can prevent the movement exceeding the range of natural movement. In hardware, the lower or upper boundary is designed for the hip joint and the knee joint respectively. The control software detects the driven current online and it will shut down the control system if the current is over the setting limit. The experiment wearing the lower extremity exoskeleton is shown in Fig. 13.

\subsection{Experimental results analysis}

Laboratory experiments were performed on healthy wearers and were carried out under the condition of safety facilities. By fastening the robotic exoskeleton with the human user, the exoskeleton can be driven to cooperate with the wearer. In the preliminary experiment, the PEM measures the pHRI, based on which, the desired control command can be obtained and sent to the actuation system. The experimental results are shown in Figs. 14-17. Figures 14 and 15 give the relationship between the PEM's signal and the estimated desired gait trajectory for the hip joint and the knee joint respectively. The desired joint trajectory has an upper bound with the safety consideration. The movement range of the hip joint is located in the interval of $(-10,40)^{\circ}$, while that of the knee joint is $(0,60)^{\circ}$. In a single gait cycle, the joint trajectory increases 


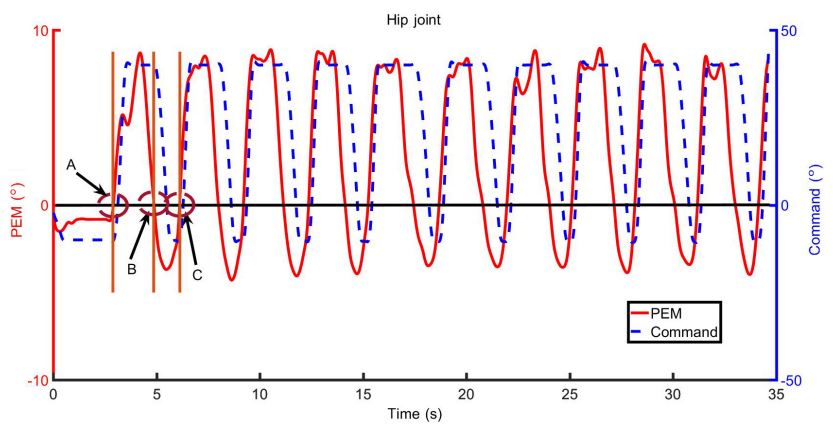

Figure 14. Relationship between the PEM signal and the command signal for the hip joint.

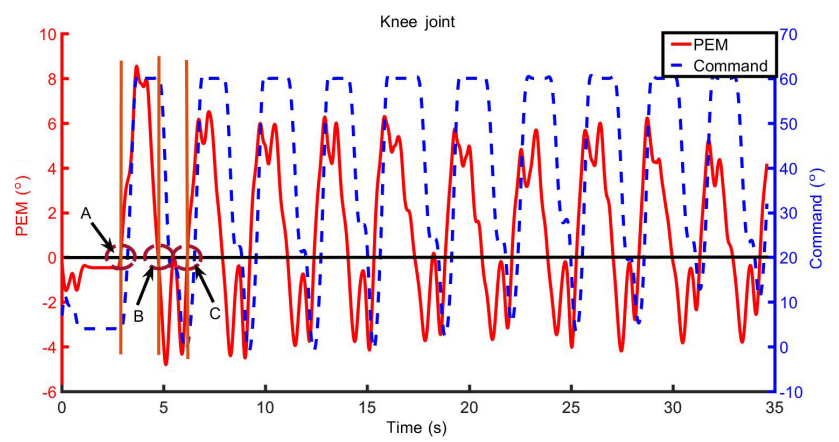

Figure 15. Relationship between the PEM signal and the command signal for the knee joint.

continuously when the PEM's signal is positive, while the joint trajectory decreases continuously when the PEM's signal is negative. In Figs. 14 and 15, these two curves between the point " $\mathrm{A}$ " and the point "B" represents the positive gait trajectory for the hip (extension) and the knee (flexion) joint respectively. Figures 16 and 17 give the gait trajectory tracking performance of the hip joint and the knee joint. In overall performance, the robotic exoskeleton can track the computed desired joint trajectory from the PEM signal quite well. In some points, the actual trajectory lags behind the command trajectory due to the higher acceleration. The experimental results in preliminary tests are used to test the system simply.

\section{Conclusions}

In this paper, a compact double elastic module is developed and characterized to be used in the lower extremity exoskeleton. The PEM is used for the pHRI collection and the DEM is applied in the elastic actuator. Compared to the traditional pHRI measurement approaches, the proposed method arranged the sensors in the mechanical joint instead of the connection cuff. This kind of architecture has compact architecture and improves the wearing comfort, which can adapt to various operators and is convenient to be applied in the wearable exoskeleton. There is only one elastic module needed

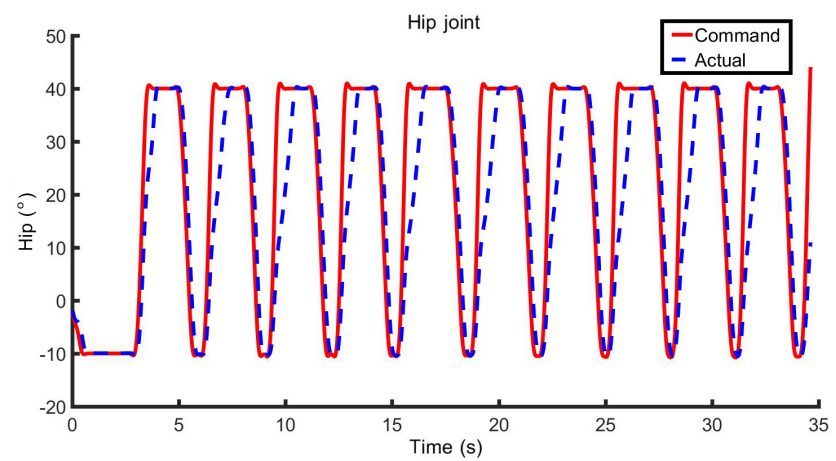

Figure 16. Trajectory tracking for the hip joint.

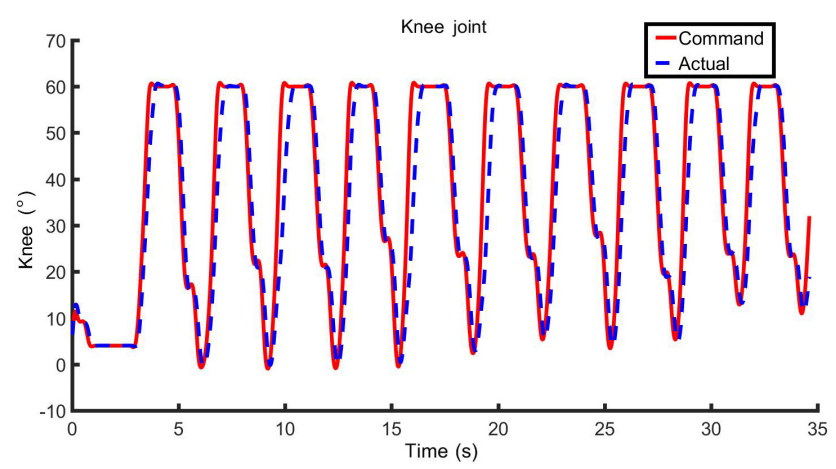

Figure 17. Trajectory tracking for the knee joint.

to measure the pHRI in the sagittal plane completely. The design and analysis process of the torsional spring is presented and calibration experiments are conducted. The PEM and the DEM have different functions and are integrated together. The preliminary experiment verifies the effectiveness of the PEM and gives the desired gait tracking performance. The performance of the DEM will be studied in the following work and human-exoskeleton coordination control strategies will be investigated in future work.

However, the work in this paper is only about the preliminary experiment for the designed robotic exoskeleton. We envision several future works to improve the human exoskeleton cooperation movement to perform motion assistance. It is worthy developing a more robust and adaptive regression algorithm for different users and improving the human exoskeleton coordination. The analysis and evaluation of the double elastic module joint will be conducted in the future work. We will pay more efforts to improve the system stability and adaptability as well as give more metabolic cost indexes to evaluate the effectiveness of the work.

Data availability. All the data used in this manuscript can be obtained by requesting from the corresponding author. 
Competing interests. The authors declare that they have no conflict of interest.

Edited by: Guangbo Hao

Reviewed by: two anonymous referees

\section{References}

Accoto, D., Carpino, G., and Sergi, F.: Design and characterization of a novel high-power series elastic actuator for a lower limb robotic orthosis, Int. J. Adv. Robot. Sys., 10, 1-10, 2013.

Arumugom, S., Muthuraman, S., and Ponselvan, V.: Modeling and application of series elastic actuators for force control multi legged robots, J. Comput., 1, 26-33, 2009.

Del-Ama, A. J., Moreno, J. C., and Gil-Agudo, A.: Online Assessment of Human-Robot Interaction for Hybrid Control of Walking, Sensors, 12, 215-225, 2012.

Dollar, A. M. and Herr, H.: Lower extremity exoskeletons and active orthoses: challenges and state-of-the-art, IEEE T. Robot., 24, 144-158, 2008.

Donati, M., Vitiello, N., and De Rossi, S. M. M.: A flexible sensor technology for the distributed measurement of interaction pressure, Sensors, 13, 1021-1045, 2013.

De Rossi, S. M. M., Vitiello, N., and Lenzi, T.: Soft artificial tactile sensors for the measurement of human-robot interaction in the rehabilitation of the lower limb, Proceedings of 2010 Annual International Conference of the IEEE Engineering in Medicine and Biology, 1279-1282, 2010a.

De Rossi, S. M. M., Vitiello, N., and Lenzi, T.: Sensing pressure distribution on a lower-limb exoskeleton physical human-machine interface, Sensors, 11, 207-227, 2010b.

Carpinom, G., Accoto, D., and Sergi, F.: A novel compact torsional spring for series elastic actuators for assistive wearable robots, J. Mech. Design, 134, 1-10, 2012.

Chen, S. Y.: Kalman filter for robot vision: a survey, IEEE T. Ind. Electron., 59, 4409-4420, 2012.

Hao, G. B.: Determinate design and analytical analysis of a class of symmetrical flexure guiding mechanisms for linear actuators, J. Mech. Design, 139, 012301, https://doi.org/10.1115/1.4034579, $2017 \mathrm{a}$.

Hao, G. B.: Determinate Synthesis of Symmetrical, Monolithic Tip-Tilt-Piston Flexure Stages, J. Mech. Design, 139, 042303, https://doi.org/10.1115/1.4035965, 2017b.

Hao, G. B., Dai, F. K., He, X. Y., and Liu, Y. F.: Design and analytical analysis of a large-range tri-symmetrical 2R1T compliant mechanism, Microsyst. Technol., 8, 1-8, https://doi.org/10.1007/s00542-017-3423-8, 2017.

Huo, W., Mohammed, S., and Moreno, J. C.: Lower limb wearable robots for assistance and rehabilitation: A state of the art, IEEE Systems Journal, 10, 1068-1081, 2016.
Kawamoto, H., Lee, S., and Kanbe, S.: Power assist method for HAL-3 using EMG-based feedback controller, in: Proceedings of IEEE International Conference on Systems, Man and Cybernetics, 2, 1648-1653, 2003.

Kazerooni, H. and Steger, R.: The Berkeley lower extremity exoskeleton, J. Dyn. Sysr.-T. ASME, 128, 14-25, 2006.

Kazerooni, H., Racine, J. L., and Huang, L.: On the control of the berkeley lower extremity exoskeleton (BLEEX), Proceedings of the 2005 IEEE International Conference on Robotics and Automation, 4353-4360, 2005.

Kong, K., Bae, J., and Tomizuka, M.: A Compact Rotary Series Elastic Actuator for Human Assistive Systems, IEEE/ASME Transactions on Mechatronics, 17, 288-297, 2012.

Lee, H. D., Yu, S. N., and Lee, S.: Development of human-robot interfacing method for assistive wearable robot of the human upper extremities, SICE Annual Conference, 1755-1760, 2008.

Lee, S. and Sankai, Y.: Power assist control for walking aid with HAL-3 based on EMG and impedance adjustment around knee joint, Proceedings of IEEE/RSJ International Conference on Intelligent Robots and Systems, 2, 1499-1504, 2002.

Lenzi, T., Vitiello, N., and De Rossi, S. M. M.: Measuring humanrobot interaction on wearable robots: A distributed approach, Mechatronics, 21, 1123-1131, 2011.

Long, Y., Du, Z. J., Wang, W. D., and Dong, W.: Development of a wearable exoskeleton rehabilitation system based on hybrid control mode, Int. J. Adv. Robot. Syst., 13, 1-10, 2016 a.

Long, Y., Du, Z. J., Wang, W. D., Zhao, G. Y., Xu, G. Q., He, L., Mao, X. W., and Dong, W.: PSO-SVM-Based Online Locomotion Mode Identification for Rehabilitation Robotic Exoskeletons, Sensors, 16, 1408, https://doi.org/10.3390/s16091408, 2016 b.

Long, Y., Du, Z. J., Cong, L., Wang, W. D., Zhang, Z. M., and Dong, W.: Active disturbance rejection control based human gait tracking for lower extremity rehabilitation exoskeleton, ISA T., 67, 389-397, 2017a.

Long, Y., Du, Z. J., Chen, C. F., Wang, W. D., He, L., Mao, X. W., Xu, G. Q., Zhao, G. Y., Li, X. Q., and Dong, W.: Development and analysis of an electrically actuated lower extremity assistive exoskeleton, J. Bionic Eng., 14, 272-283, 2017 b.

Sankai, Y: HAL: Hybrid assistive limb based on cybernics, Robotics Research, Springer Berlin Heidelberg, 25-34, 2010.

Santis, A. D., Siciliano, B., and Luca, A. D.: An atlas of physical human-robot interaction, Mech. Mach. Theory, 43, 253-270, 2008.

Veneman, J. F., Kruidhof, R., and Hekman, E. E. G.: Design and evaluation of the LOPES exoskeleton robot for interactive gait rehabilitation, IEEE T. Neur. Sys. Reh., 15, 379-386, 2007.

Yoshimitsu, T. and Yamamoto, K.: Development of a power assist suit for nursing work, Proceedings of 2004 SICE Annual Conference, 1, 577-580, 2004. 